\title{
CSAP - AN ADAPTABLE SECURITY MODULE FOR THE E-GOVERNMENT SYSTEM WEBOCRAT
}

\author{
Fredj Dridi, Michael Fischer and Günther Pernul \\ Department of Information Systems, \\ University of Regensburg \\ Universitätsstr. 31, D-93053 Regensburg, Germany \\ \{fredj.dridi,guenther.pernul\} @wiwi.uni-regensburg.de, michael.fischer@gmx.de
}

\begin{abstract}
In this paper we present a generic and adaptable security module called CSAP (Communication, Security, Authentication, and Privacy) which may be used in order to make e-government and e-commerce systems secure and trustworthy. CSAP is service-oriented and offers programming interfaces to core security services such as user identification, authentication, access control, auditing, and security management. We discuss the conceptual architecture, the layered design, and the object-oriented implementation of CSAP. The layered design of CSAP allows the application developer to exchange or enhance security mechanisms via a plug-in concept based on abstract classes and appropriate design patterns. As a consequence, CSAP becomes maintainable and adaptable.

Among other services, CSAP provides an implementation of a rolebased access control subsystem that conforms to the Core RBAC model as defined in the proposed NIST standard. Currently, CSAP is almost fully implemented and integrated in the WEBOCRAT system which in the framework of e-government - provides services supporting direct participation of citizens in democratic processes in a secure way.
\end{abstract}

Keywords: Security architecture and services, adaptable security systems, software design, role-based access control (RBAC).

\section{Introduction}

E-government is the term reflecting the use of Information and Communication Technologies in public administration in an attempt to ease access to governmental information and services for citizens, businesses, and government agencies. Furthermore it is always a target to improve the quality of the services and to provide greater opportunities for participating in democratic institutions and processes.

The original version of this chapter was revised: The copyright line was incorrect. This has been corrected. The Erratum to this chapter is available at DOI: 10.1007/978-0-387-35691-4_52 
It is essential for e-government and e-commerce that information and services are adequately protected and that the citizen's/customer's privacy is maintained. Sensitive government, business, and citizen data need to be fully secured when stored, processed or transmitted across institutional, regional, national, and possibly international networks. The problem of developing security policies and services in such environments becomes critical [Joshi et al., 2001]. Today developers are faced with building (web-based) systems to serve an unknown, large number of concurrent users with a virtually unpredictable usage patterns. As a result, these systems must deploy a security architecture providing security services which are easily adaptable to changing security requirements.

In this work, we present a generic security architecture and its design and Java implementation. The implementation is called CSAP. This has been done within the context of the EU-funded Webocracy ${ }^{1}$ project, whose aim is the innovative use of state-of-the-art web technologies in order to support direct participation of citizens in democratic processes [Dridi et al., 2001; Paralic et al., 2002]. In this project a system called WEBOCRAT is modularly designed and implemented. The WEBOCRAT system is an e-government system focusing on functions like discussion management, publishing on the web, opinion polling, intelligent retrieval of information, reporting and limited public procurement.

CSAP is an integral module within the WEBOCRAT system and its system requirements are specified by our user partners (three local authorities). However, CSAP is designed in a generic way so that it can be used by other types of web-based applications as well. Currently, CSAP is almost fully implemented and integrated in the WEBOCRAT system to make it secure and trustworthy. The key features of CSAP are: (a) The CSAP architecture is service-oriented, providing just the right level of abstraction and encapsulation to fulfill user requirements. (b) The layered design of CSAP allows the application developer to exchange or enhance security mechanisms via a plug-in concept based on abstract classes and appropriate design patterns [Gamma et al., 1995]. As a consequence, CSAP becomes maintainable and adaptable. (c) The CSAP module is easily deployable via standard Java technology (e.g., JSP/Servlets). (d) Among other services, CSAP implements a rolebased access control subsystem that conforms to the Core RBAC model as defined in the proposed NIST standard [Ferraiolo et al., 2001].

These features of CSAP allow system designers to implement manageable and extensible security platforms. Such platforms can be deployed

\footnotetext{
${ }^{1}$ The project's full title is "Web Technologies Supporting Direct Participation in Democratic Processes". The acronym "Webocracy" means "Democracy on the Web".
} 
to experiment flexibly with emerging "pluggable" security services, as well as to build scalable and secure web-based applications. This claim will be justified in the remainder of this paper which is structured as follows. Section 2 presents a generic service-oriented security architecture, the key security services and the requirements concerning its realization. Section 3 presents the layered design, implementation and use scenarios of CSAP. The presented use scenarios, focusing on the user authentication and access control processes, demonstrate the integration of CSAP into web-based applications. Section 4 discusses related work and Section 5 concludes the paper and gives an outlook on future work.

\section{Generic Security Architecture}

Generally, the following basic security objectives have been determined for web-based application (for example, see [Dridi and Neumann, 2000]): confidentiality, integrity, availability, authenticity of data, nonrepudiation, proof of identification. Figure 1 illustrates the service-

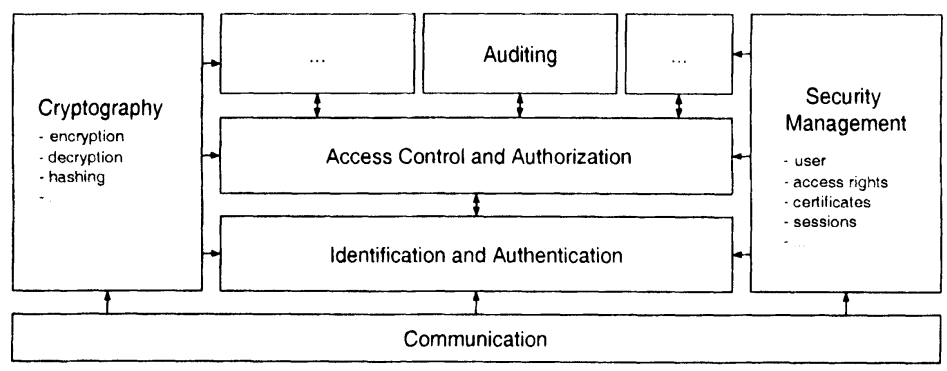

Figure 1. Generic, Service-Oriented Security Architecture. Necessary key security services to be provided and their interdependencies.

oriented security architecture we identified for achieving these security objectives. It is generic and service-oriented in the sense that it provides standard security services for a large array of applications.

\subsection{Security Services}

The key security services are: (1) Identification and Authentication is needed in order to get and verify the identity of the users attempting to access the system. (2) Access Control and Authorization is needed in order to verify what actions the users are able to perform and what information the users are able to access. (3) Auditing is needed to log all security relevant actions in order to make users accountable for their transactions. Audit trails are used to find patterns of abnormal use 
which are often a sign of (attempted) compromise. (4) Security Management is needed to manage (create, update, storage, etc.) global security information used within applications. Such management services are essential in order to customize and administrate security for applications.

All these services may require standard cryptographic services (such as encryption, decryption, hashing, etc.) and communication services. Such services are usually provided by existing software packages (e.g. Java 2 Platform) and therefore they do not need to be implemented again. The focus of our work is the usable integration of the key security services. As a consequence, several key realization requirements have

\section{$2.2 \quad$ Realization Requirements}

Several security systems implementing the generic security architecture discussed above have been built, but they all have their own restrictions that often do not correspond with the specific requirements of e-commerce and e-government applications (compare [Win et al., 2000], for a recent discussion of requirements [Lambrinoudakis et al., 2002]). Therefore, we need to implement an adaptable security system which can be deployed in different environments. In our case, the internationally dispersed project partners imposed different requirements on CSAP (the implementation of the generic security architecture) (Paralic and Sabol, 2001]. For example, the cooperating local authority from UK plans to use a PKI which will be developed to build secure forms of user authentication, including digital signatures. The Slovak local authorities want to use a user-password scheme. All the user partners have agreed to use RBAC. But we can imagine that, if CSAP will be used within another environment, different access control services (e.g. using access control lists) may be required. As a result the need to design a system where the security services are completely changeable is identified. The solution we provide is "pluggable" security services (to be explained below).

In order to realize interoperation with underlying backend systems it may be required that existing data (e.g. user data), which are relevant for the security system, have to be used regardless where it is stored. This way the decision about the storage system (files, databases, directory services) must not be hard-coded and has to remain configurable. Furthermore, security systems have to be application independent and scalable.

In order to achieve these requirements we deployed state-of-the-art software engineering techniques. We used appropriate design patterns [Gamma et al., 1995], UML (Unified Modeling Language [Booch et al., 1999]) specifications and the object-oriented language Java. Next we 
describe our CSAP module in more detail and show how those techniques have been used.

\section{The CSAP Module}

During the development of CSAP our overall goal was to develop a general, flexible and extensible module in which applications can selectively and dynamically access security services. As security requirements in large scale systems are changing constantly, CSAP should allow the developer to integrate new security services or to enhance existing ones. This should not require modification, recompilation, or even notification of client code unless there is a significant change in the specification. The term "client" refers to a user request or to any WEBOCRAT module which wants to use the CSAP services. For example, a CSAP client may be the Discussion module requesting user authentication or the Publishing module requesting access to a sensitive document.

\subsection{Design}

Figure 2 depicts the layered design of CSAP. It offers separation of concerns, because we can deal with parts of the source code in isolation, with minimal or no side effects in other layers. The design consists of three layers:

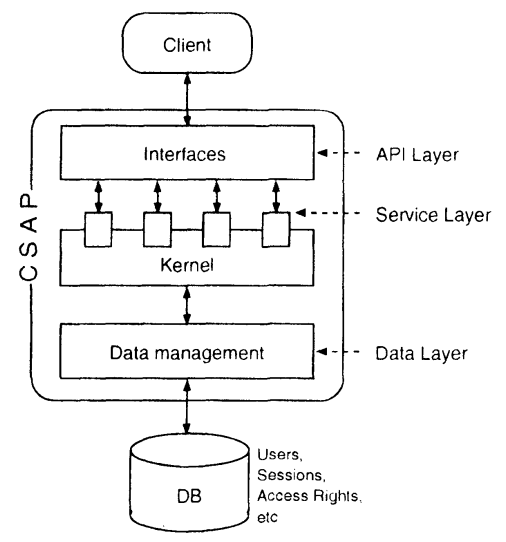

Figure 2. CSAP Layered Design.

The API Layer provides unified access to the security services implemented by CSAP. This layer separates the usage of a given security service from its implementation. Applications request security services only through the CSAP API. 
The Service Layer is built upon the kernel of CSAP and provides the infrastructure for changing an existing security service or for plugging-in new services. All the security services discussed above are integrated in this layer. The clients use a service via the API layer. The data needed by the service are provided by the data layer.

The Data Layer provides the flexibility to access and manage user security information (e.g. access rights, passwords, session-ids, etc.). This layer defines a generic interface in order to access the security information independent of how they are stored. In CSAP the security information may be stored in XML files, in LDAP directory services [Wahl et al., 1997], or in SQL databases (e.g. MySQL). How these data is actually stored depends on the application and can be configured within CSAP. The data layer uses the security services implemented in CSAP to avoid non-authorized modifications of the security inforamtion. In other words the access to the CSAP databases is controlled with CSAP itself.

An example should illustrate the functionality of the layered CSAP design: Consider the RBAC service. On the API Layer, the authenticated user requests a certain role from the RBAC service at the Service Layer. Based on the role data accessed on the Data Layer the RBAC service presents the set of available roles. After the user has chosen a certain role, the RBAC service verifies, based on the permission data from the Data Layer, whether the user is allowed to activate the role. In case he is allowed the corresponding permissions are derived from the Data Layer.

By using such a layered design, we made CSAP adaptable so that we can easily exchange or enhance the security services according to changing requirements. The service and the data layer are configured by using an XML file. The structure of this configuration file is simple and will not be discussed here. In the next section we focus on the implementation issues of the authentication (user-password scheme) and access control services (Core RBAC).

\subsection{Implementation Issues}

The API Layer is implemented by means of Java interfaces and the design pattern "Facade". The concept of a Facade [Gamma et al., 1995] was chosen in order to hide the complexity of the CSAP security services from the clients by presenting a high-level, easy-to-use interface to them. This way a clear separation of implementation and usage aspects of the security services is achieved. The facade pattern forces the clients to use a single entry point to perform in an uniform way the calls to the security 
services. Due to space limitations this will not be described in this paper. The Data Layer is modeled by using the design pattern "Abstract

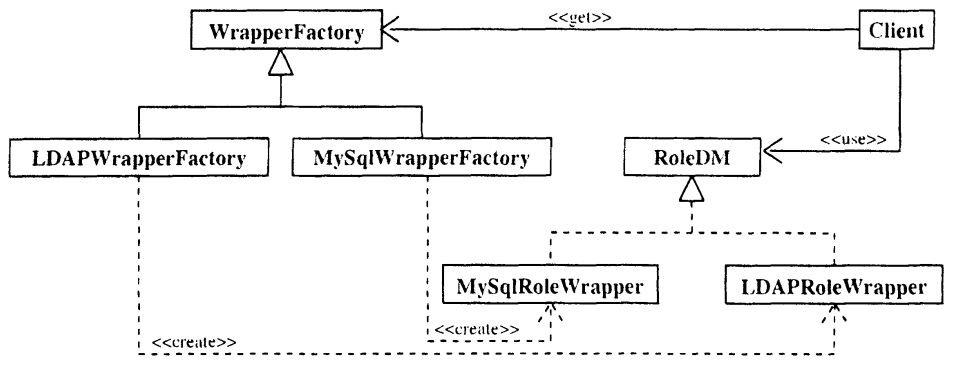

Figure 3. Abstract Factory within CSAP. A client is able to access data regardless of where it is stored. The WrapperFactory creates concrete wrapper implementing the access methods to the used database. The RoleDM is responsible for managing role data.

Factory" [Gamma et al., 1995]. The purpose of the Abstract Factory is to provide a generic interface for creating families of related wrapper objects without specifying their concrete classes, compare Figure 3.

Figure 4 depicts the core classes implementing the Service Layer and the kernel of CSAP. The abstract classes shown in this figure form the kernel of CSAP. We distinguish two kinds of classes: service classes and product classes. Service classes are responsible for creating products. A product is an instance of a class modeling low level objects within CSAP, like User, Object, Operation, Permission, Session and Role. The common service and product properties are defined in the classes AbstractService and AbstractProduct respectively. The CSAP clients mainly use the product classes User and Session. The remaining product classes are used for administrating the Core RBAC system. Basically, a CSAP client gets products from the corresponding service classes and uses the interfaces the products implement. The user/password service and the Core RBAC service are implemented within the two service classes PasswordService and RBACService respectively. The password service mainly implements a login method and creates an authenticated user object which can be used, for example, by the RBAC service. The RBAC service of CSAP implements a checkAccess method which determines whether an access is allowed or denied. In addition, it implements all the necessary methods as proposed by NIST to administer the RBAC system. This functionality is used for a servlet-based implementation of the CSAP administration console, which in itself is a GUI to administer RBAC. 


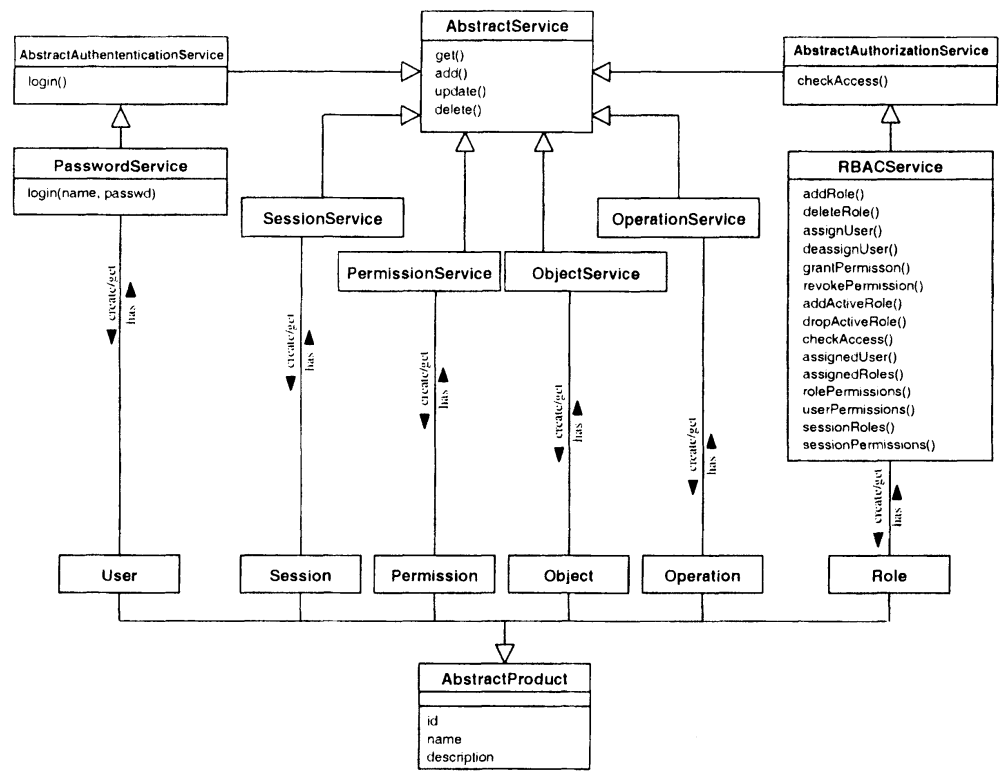

Figure 4. CSAP Service and Product Classes. New security services can be implmented by subclassing the corresponding absract classes.

CSAP can be tailored to different situations in which other security services may be needed. For example, in order to implement a certificatebased authentication service, a new service class (e.g. CertService) has to be defined as a subclass of the class AbstractAuthenticationService. This new service class could be used alternatively to the PasswordService. Both classes AbstractAuthenticationService and AbstractAuthorizationService can be used to define new subclasses implementing alternative authentication and access control mechanisms respectively.

\subsection{Use Scenario}

In this section, we describe how to use the CSAP module focusing on authentication and access control. Assume a situation in which CSAP is configured to use both the PasswordService and the RBACService. The authentication process consists of the following steps:

- Get plugged services: A request starts with a client asking the CSAP system which kind of authentication and access control services are available. In this example the client gets a reference to the PasswordService and RBACService. 
- Login: The PasswordService implements a login method which accepts a username/password as parameters. If the authentication process is successful a user object will be created.

- Select roles: Due to the RBAC policy at least one role has to be selected as active role. The RBACService and the RoleDM (see Figure 3) will determine the set of the roles assigned to the user. The active role specifies the set of permissions a user may posses.

- Create session: As a result of the client request, a session object will be created in order to store the user object and the activated role for further use. After a session is established the session data are stored by the SessionDM. Sessions usually have a limited life time, after this time a session becomes invalid and will be deleted automatically by the SessionDM.

Before an access can be checked the user must be correctly authenticated and a valid session object must exist. In the example shown in Figure 5 we assume that the above sequence has been performed and that the client holds a valid session-id. The access control process is determined as follows:

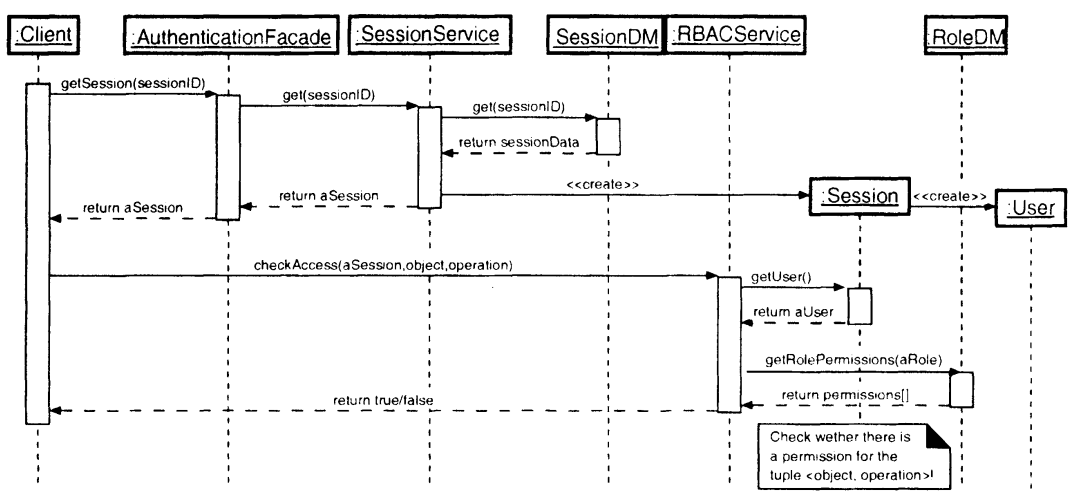

Figure 5. Sequence Diagram - Role-based Access Control.

- Use Session: The client gets the session object from the SessionService using the assigned session-id. If the session is no longer valid, the user has to be asked by the client to re-authenticate and thereby to establish a new valid session.

- Check Access: The method checkAccess accepts three arguments: a session-id, operation name and object name. The user object 
that is stored within the session object is used by the RBACService in order to get the user's active role within this session. The user's permissions are determined by retrieving all the permissions assigned to the active role. Finally, the RBACService checks whether there is a permission for the tuple <object,operation>. In the positive case, the access will be granted otherwise denied.

\section{Related Work}

In this section we discuss some related work which have influenced the design and implementation of the CSAP module.

In [Park et al., 2001], Sandhu et al. present several implementation approaches for role-based access control. For this purpose well-known technologies with smart extensions such as cookies, X.509 certificates, SSL, and LDAP, providing compatibility with current Web technologies, have been used. For example, X.509 certificates have been extended in order to include the roles assigned to a particular user. In contrast to the CSAP approach their work is mainly restricted to a singular access control service only and not embedded into a large scale application.

Gutzmann [Gutzmann, 2001] introduces a security service architecture for HTTP-based environments. He describes an approach that uses RBAC and Web session management to prevent network security breaches. He uses LDAP and a ticket-based management service to realize an RBAC service. His work is focusing on session management, with CSAP we take a broader approach.

The Java Authentication and Authorization Service (JAAS) [Lai et al., 1999] augments JDK with support to authenticate the principal who runs the code and to enforce new access controls on the authenticated principal. It provides a flexible control of downloaded executable content and it has been extended with roles based on Java Servlets in [Giuri, 1999]. Although related to our work, their focus is mainly on mobile code and mobile agent authorization. Moreover, their focus was on extending the Java functionality while CSAP is building on the existing standard.

The Pluggable Authentication Modules (PAMs) [Samar and Lai, 1996] provide a well structured architecture for the integration of multiple authentication mechanisms. By using PAM, users who need access to certain network resources or who $\log$ in from outside the firewall may utilize strong authentication mechanisms. The main advantage of PAM is that administrators are able to select one or multiple authentication technologies without modifying applications or utilities. This is similar to our approach, however, CSAP is not realized at the operating system level nor does it depend on certain operating systems. 


\section{Conclusion}

We have presented the architecture of CSAP, an autonomous software module offering programming interfaces to core security services such as authentication, access control, auditing, and security management. It is currently developed as part of the ongoing EU-funded Webocracy project which - in the framework of e-government - provides services supporting direct participation of citizens in democratic processes in a secure way.

The key design objective adaptability has resulted in a layered design including an Application Programming Interface Layer, a Service Layer and a Data Layer. The Application Programming Interface Layer provides unified access to the security services. At the Service Layer, different security mechanisms can be changed by using an easy-to-use plug-in technique. The Data Layer provides the flexibility to access and manage user security information independent of the storage platform. The current implementation of CSAP provides a password service subsystem and a role-based access control subsystem that conforms to the Core $R B A C$ model as defined in the proposed NIST standard. CSAP is expected to provide a viable framework for addressing a wide range of security requirements for large scale applications like WEBOCRAT.

CSAP is available as a platform for implementing or extending existing security services, for experimenting with different security service alternatives, and for building secure and manageable large scale webapplication. Our experience has shown that the chosen software design of CSAP is suitable for server-side integration.

In future work, the implementation will be extended to include alternative plug-in security services, like certificate-based authentication and access control. A graphical (web-based) user interface to the RBAC service within CSAP is currently under development. In addition we will improve the configuration functionality of CSAP to manage several applications with different storage requirements. Finally, after CSAP will have been tested in real settings by the user partners, some optimisations (e.g. performance) will be considered.

\section{Acknowledgments}

We would like to thank Wolfram Conen and our project partners for helpful comments and stimulating discussions. This work is done within the Webocracy Project which is supported by European Commission DG INFSO under the IST programme, contract No. IST-1999-20364. 


\section{References}

Booch, G., Rumbaugh, J., and Jacobson, I. (1999). The Unified Modeling Language Reference Manual. Addison Wesley.

Dridi, F. and Neumann, G. (2000). Managing security in the World Wide Web: Architecture, services, and techniques. In Janczewski, L., editor, Internet and Intranet Security Management: Risks and Solutions, pages 106-139. Hershey PA: Idea Group Publishing.

Dridi, F., Pernul, G., and Sabol, T. (2001). The Webocracy project: Overview and security aspects. In Schnurr, H.-P., S. Stabb, R. Studer, G. S., and Sure, Y., editors, Professionelles Wissensmanagement: Erfahrungen und Visionen, pages 401-408, Aachen, Germany. Shaker Verlag.

Ferraiolo, D., Sandhu, R., Gavrila, S., Kuhn, D., and Chandramouli, R. (2001). Proposed NIST standard for role-based access control. ACM Transactions on Information and Systems Security, 4(3):224-274.

Gamma, E., Helm, R., Johnson, R., and Vlissides, J. (1995). Design Patterns: Elements of Reusable Object-Oriented Software. Addison-Wesley.

Giuri, L. (1999). Role-based access control on the web using java. In Proceedings of the 4th ACM workshop on Role-based access control, pages 11-18, Fairfax, Virginia.

Gutzmann, K. (2001). Access control and session management in the http environment. IEEE Internet Computing, 5(1):26-35.

Joshi, J., Ghafoor, A., Aref, W., and Spafford, E. (2001). Digital government security infrastructure design challenges. Computer, 34(2).

Lai, C., Gong, L., Koved, L., Nadlin, A., and Schemers, R. (1999). User authentication und authorization in the java platform. In Proceedings of the 15th Annual Computer Security Application Conference, pages 285-290, Phoenix, Arizona.

Lambrinoudakis, C., Gritzalis, S., Dridi, F., and Pernul, G. (2002). Security requirements for e-government services: A methodological approach for developing a common PKI- based security policy. Comupter Communications, Special Issue on PKI.

Paralic, J. and Sabol, T. (2001). Implementation of e-government using knowledgebased system. In Proc. of the 2nd Int. Workshop on Electronic Government within DEXA 2001, pages 364-368, Munich, Germany.

Paralic, J., Sabol, T., and Mach, M. (2002). A system to support e-democracy. In Proc. of the 1st eGovernment Conference within DEXA2002, pages 288-291, Aixen-Provence, France.

Park, J., Sandhu, R., and Ahn, G. (2001). Role-based access control on the web. ACM Transactions on Information and Systems Security, 4(1).

Samar, V. and Lai, C. (1996). Making login services independent of authentication technologies. In Proceedings of the 3rd ACM Conference on Computer Communications and Security.

Wahl, M., Howes, T., and Kille, S. (1997). Lightweight directory access protocol (v3). RFC 2251, Standards Track.

Win, B. D., den Bergh, J. V., Matthijs, F., Decker, B. D., and Joosen, W. (2000). A security architecture for electronic commerce applications. In IFIP TC11 16th Annual Working Conference on Information Security: Information Security for Global Information Infrastructures, pages 491-500, Beijing, China. 